\title{
Modulated structures in the ANNNI model with an external magnetic field near criticality
}

\author{
N.S.Gonchar, H.G.Hajduk \\ Bogolubov Institute for Theoretical Physics \\ of the National Academy of Sciences of Ukraine, \\ $14^{6}$ Metrolohichna Str., 252143 Kyiv, Ukraine
}

Received December 3, 1997

Conditions for a phase transition from the paramagnetic state to the modulated structure are found in a class of anisotropic Ising models with an external magnetic field. The critical value of the external magnetic field is obtained. Branching equations are derived and small branching theorems are proven for commensurate and incommensurate configurations.

Key words: anisotropic Ising model, phase transition, paramagnetic phase, commensurate configuration, incommensurate configuration, critical magnetic field

PACS: $05.50 .+q, 64.60 . C n$

Here we consider a class of anisotropic Ising models with an external magnetic field and show a phase transition from the paramagnetic state to spatially inhomogeneous commensurate configuration or incommensurate one below the critical magnetic field. To be more exact, we illustrate the method on the ANNNI model $[1,2]$, but one can apply it to models with an arbitrary number of interacting neighbours.

Any $N_{1}$-periodic configuration $x$ can be expressed [3] as

$$
x=\sum_{l=1}^{N}\left(a_{l} e_{1}(l q)+b_{l} e_{2}(l q)\right)+a_{0} \epsilon
$$

where $N=\left[N_{1} / 2\right],[c]$ is an integral part of $c, q=m / N_{1}$ is an irreducible quotient,

$$
\begin{gathered}
\epsilon=\left\{a_{i}\right\}_{i=-\infty}^{+\infty}, a_{i}=1 \quad \forall i \\
e_{1}(q)=\{\cos i 2 \pi q\}_{i=-\infty}^{+\infty}, e_{2}(q)=\{\sin i 2 \pi q\}_{i=-\infty}^{+\infty} .
\end{gathered}
$$


Then, the specific free energy for commensurate configuration (1) has the form [4]

$$
\begin{aligned}
F_{h}=- & \frac{1}{2}\left(\frac{1}{2} \sum_{l=1}^{N}\left(\left(1+\delta_{l,\left[\frac{N_{1}+1}{2}\right]}\right) a_{l}^{2}+\left(1-\delta_{l,\left[\frac{N_{1}+1}{2}\right]}\right) b_{l}^{2}\right) \Phi(l q)\right. \\
& \left.+a_{0}^{2} \Phi(0)\right)+h a_{0}+\frac{1}{2} T\langle\epsilon, g(x)\rangle,
\end{aligned}
$$

where function $g$ is

$$
\begin{aligned}
g(x) & =(1+x) \ln (1+x)+(1-x) \ln (1-x) \\
& =\lim _{n \rightarrow \infty} 2\left(\frac{x^{2}}{2}+\frac{x^{4}}{3 \cdot 4}+\cdots+\frac{x^{2 n}}{2 n \cdot(2 n-1)}\right)
\end{aligned}
$$

provided that

$$
\sum_{l=1}^{N}\left(\left|a_{l}\right|+\left|b_{l}\right|\right)+\left|a_{0}\right|<1
$$

For the ANNNI model

$$
\Phi(q)=-2\left(J_{1} \cos 2 \pi q+J_{2} \cos 4 \pi q+2 J_{0}\right),
$$

and for the case when all the neighbours interact

$$
\Phi(q)=-2\left(\sum_{i=1}^{+\infty} J_{i} \cos 2 \pi \mathrm{i} q+2 J_{0}\right) .
$$

The free energy (2) is invariant under a discrete transformation group [5]

$$
\begin{gathered}
a_{l}^{\prime}=\quad a_{l} \cos 2 \pi l s q+b_{l} \sin 2 \pi l s q, \\
b_{l}^{\prime}=-a_{l} \sin 2 \pi l s q+b_{l} \cos 2 \pi l s q, \\
q=m / N_{1}, \quad s=0,1, \cdots, N_{1} .
\end{gathered}
$$

The free energy of incommensurate configuration (1) with irrational $q$ and arbitrary $N$ is [6]

$$
F_{h}=-\frac{1}{2}\left(\frac{1}{2} \sum_{l=1}^{N}\left(a_{l}^{2}+b_{l}^{2}\right) \Phi(l q)+a_{0}^{2} \Phi(0)\right)+h a_{0}+\frac{1}{2} T\langle\epsilon, g(x)\rangle .
$$

The free energy (4) is invariant of group $G[3]$

$$
\begin{gathered}
a_{l}^{\prime}=a_{l} \cos l \phi_{0}+b_{l} \sin l \phi_{0}, \\
b_{l}^{\prime}=-a_{l} \sin l \phi_{0}+b_{l} \cos l \phi_{0}, \\
\phi_{0} \in[0,2 \pi] .
\end{gathered}
$$


The necessary conditions of a free energy minimum are the equations

$$
\begin{aligned}
& -a_{l} \Phi(l q)\left(1+\delta_{l,\left[\frac{N_{1}+1}{2}\right]}\right)+T\left\langle\epsilon, g^{\prime}(x) e_{1}(l q)\right\rangle=0, \\
& -b_{l} \Phi(l q)\left(1-\delta_{l,\left[\frac{N_{1}+1}{2}\right]}\right)+T\left\langle\epsilon, g^{\prime}(x) e_{2}(l q)\right\rangle=0 \\
& -a_{0} \Phi(0)+h+T\left\langle\epsilon, g^{\prime}(x)\right\rangle / 2=0, \\
& g^{\prime}(x)=\ln \frac{1+x}{1-x}=2\left(x+\frac{x^{3}}{3}+\frac{x^{5}}{5}+\cdots+\frac{x^{2 n+1}}{2 n+1}+\cdots\right),
\end{aligned}
$$

for rational $q$ and

$$
\begin{aligned}
-a_{l} \Phi(l q)+T\left\langle\epsilon, g^{\prime}(x) e_{1}(l q)\right\rangle & =0 \\
-b_{l} \Phi(l q)+T\left\langle\epsilon, g^{\prime}(x) e_{2}(l q)\right\rangle & =0 \\
-a_{0} \Phi(0)+h+T\left\langle\epsilon, g^{\prime}(x)\right\rangle / 2 & =0
\end{aligned}
$$

for irrational $q$.

In the external magnetic field the systems (6) and (7) have a nonzero solution in the set of vectors $\left\{\left(a_{1}, b_{1}\right), \cdots,\left(a_{N}, b_{N}\right), a_{0}\right\}$ such that

$$
\sum_{l=1}^{N}\left(\left|a_{l}\right|+\left|b_{l}\right|\right)+\left|a_{0}\right|<1 .
$$

The solution has the form $\left\{(0,0), \cdots,(0,0), \bar{a}_{0}\right\}$ with $\bar{a}_{0}$ solving the equation

$$
-\bar{a}_{0} \Phi(0)+T g^{\prime}\left(\bar{a}_{0}\right) / 2+h=0 .
$$

We assume that

$$
\begin{aligned}
x & =\bar{x}+\bar{a}_{0} \epsilon \\
\bar{x} & =\sum_{l=1}^{N}\left(a_{l} e_{1}(l q)+b_{l} e_{2}(l q)\right)+a_{0}^{\prime} \epsilon \\
g^{\prime}(x) & =\sum_{k=1}^{+\infty} \frac{g^{(k)}\left(\bar{a}_{0}\right)}{(k-1) !} \bar{x}^{k-1}=g^{\prime}\left(\bar{a}_{0}\right) \epsilon+g^{\prime \prime}\left(\bar{a}_{0}\right) \bar{x}+\varphi(\bar{x}), \\
g^{\prime}\left(\bar{a}_{0} \epsilon\right) & =g^{\prime}\left(\bar{a}_{0}\right) \epsilon, \quad \bar{x} \epsilon=\bar{x} \\
\varphi(\bar{x}) & =\sum_{k=3}^{+\infty} \frac{g^{(k)}\left(\bar{a}_{0}\right)}{(k-1) !} \bar{x}^{k-1} .
\end{aligned}
$$

Then, system (6) becomes

$$
\begin{aligned}
a_{l}\left(T /\left(1-\bar{a}_{0}^{2}\right)-\Phi(l q)\right)\left(1+\delta_{l,\left[\frac{N_{1}+1}{2}\right]}\right)+T\left\langle\epsilon, \varphi(\bar{x}) e_{1}(l q)\right\rangle & =0 \\
b_{l}\left(T /\left(1-\bar{a}_{0}^{2}\right)-\Phi(l q)\right)\left(1-\delta_{l,\left[\frac{N_{1}+1}{2}\right]}\right)+T\left\langle\epsilon, \varphi(\bar{x}) e_{2}(l q)\right\rangle & =0 \\
2 a_{0}^{\prime}\left(T /\left(1-\bar{a}_{0}^{2}\right)-\Phi(0)\right)+T\langle\epsilon, \varphi(\bar{x})\rangle & =0
\end{aligned}
$$


and system (7) becomes

$$
\begin{aligned}
a_{l}\left(T /\left(1-\bar{a}_{0}^{2}\right)-\Phi(l q)\right)+T\left\langle\epsilon, \varphi(\bar{x}) e_{1}(l q)\right\rangle & =0 \\
b_{l}\left(T /\left(1-\bar{a}_{0}^{2}\right)-\Phi(l q)\right)+T\left\langle\epsilon, \varphi(\bar{x}) e_{2}(l q)\right\rangle & =0 \\
2 a_{0}^{\prime}\left(T /\left(1-\bar{a}_{0}^{2}\right)-\Phi(0)\right)+T\langle\epsilon, \varphi(\bar{x})\rangle & =0
\end{aligned}
$$

A spatially inhomogeneous solution of systems (6), (7) exists if systems (9), (10) have a nonzero solution. The first degeneration of the spectrum of the linearized part of nonlinear operator (9) or (10) occurs when

$$
\begin{aligned}
T /\left(1-\bar{a}_{0}^{2}\right)-\Phi(q) & =0 \\
-\bar{a}_{0} \Phi(0)+T g^{\prime}\left(\bar{a}_{0}\right) / 2+h & =0 .
\end{aligned}
$$

If there exists a solution to system (11), then the zero solution to systems (9), (10) bifurcates. It means that a transition from the paramagnetic phase to the modulated structure occurs. As a result, system (11) gives a single equivalent equation

$$
T=\Phi(q)-\Phi(q) h^{2} /\left[\Phi(0)-\frac{1}{2} T(1-T / \Phi(q))^{-\frac{1}{2}} g^{\prime}(1-T / \Phi(q))^{\frac{1}{2}}\right]^{2} .
$$

From (12) it follows:

Lemma 1. The critical magnetic field for a phase transition from the paramagnetic state to the modulated structure is equal to $|\Phi(0)|$.

For the proof see [7].

Having in mind that the mean-field theory critical exponent $\beta$ equals $1 / 2$, we suppose a deviation from the critical temperature to be standard. Therefore,

$$
\lambda^{2}(T, h)=\Phi(q)-T /\left(1-\bar{a}_{0}^{2}\right),
$$

where $\bar{a}_{0}$ solves equation (8). If $T_{0}(h)$ solves equation $(12)$, then $\lambda^{2}\left(T_{0}(h), h\right)$ equals zero.

In terms of new variables, system (10) for irrational $q$ takes the form:

$$
\begin{gathered}
-\lambda^{2} a_{1}+T\left\langle\epsilon, \varphi(\bar{x}) e_{1}(q)\right\rangle=0, \\
-\lambda^{2} b_{1}+T\left\langle\epsilon, \varphi(\bar{x}) e_{2}(q)\right\rangle=0, \\
a_{l}\left(\Phi(q)-\Phi(l q)-\lambda^{2}\right)+T\left\langle\epsilon, \varphi(\bar{x}) e_{1}(l q)\right\rangle=0, \\
b_{l}\left(\Phi(q)-\Phi(l q)-\lambda^{2}\right)+T\left\langle\epsilon, \varphi(\bar{x}) e_{2}(l q)\right\rangle=0, \\
2 a_{0}^{\prime}\left(\Phi(q)-\Phi(0)-\lambda^{2}\right)+T\langle\epsilon, \varphi(\bar{x})\rangle=0 .
\end{gathered}
$$

We seek the solution of systems (13), (14) in the form

$$
a_{1}=\lambda \tilde{a}_{1}, \quad b_{1}=\lambda \tilde{b}_{1}, \quad a_{l}=\lambda^{2} \tilde{a}_{l}, \quad b_{l}=\lambda^{2} \tilde{b}_{l}, \quad a_{0}^{\prime}=\lambda^{2} \tilde{a}_{0}^{\prime} .
$$


Then we rewrite (13), (14) as

$$
\begin{gathered}
\tilde{a}_{1}=F_{1}^{(1)}(\tilde{w})=\frac{T}{\lambda^{3}} \sum_{k=3}^{+\infty} \frac{g^{(k)}\left(\bar{a}_{0}\right)}{(k-1) !} \lambda^{k-1}\left\langle\epsilon,(\cdot)^{k-1} e_{1}(q)\right\rangle, \\
\tilde{b}_{1}=F_{1}^{(2)}(\tilde{w})=\frac{T}{\lambda^{3}} \sum_{k=3}^{+\infty} \frac{g^{(k)}\left(\bar{a}_{0}\right)}{(k-1) !} \lambda^{k-1}\left\langle\epsilon,(\cdot)^{k-1} e_{2}(q)\right\rangle \\
\tilde{a}_{l}=F_{l}^{(1)}(\tilde{w})=\frac{T}{\lambda^{2}\left(\lambda^{2}+\Phi(l q)-\Phi(q)\right)} \sum_{k=3}^{+\infty} \frac{g^{(k)}\left(\bar{a}_{0}\right)}{(k-1) !} \lambda^{k-1}\left\langle\epsilon,(\cdot)^{k-1} e_{1}(l q)\right\rangle, \\
\tilde{b}_{l}=F_{l}^{(2)}(\tilde{w})=\frac{T}{\lambda^{2}\left(\lambda^{2}+\Phi(l q)-\Phi(q)\right)} \sum_{k=3}^{+\infty} \frac{g^{(k)}\left(\bar{a}_{0}\right)}{(k-1) !} \lambda^{k-1}\left\langle\epsilon,(\cdot)^{k-1} e_{2}(l q)\right\rangle, \\
\tilde{a}_{0}^{\prime}=F_{0}^{(0)}(\tilde{w})=\frac{T}{2 \lambda^{2}\left(\lambda^{2}+\Phi(0)-\Phi(q)\right)} \sum_{k=3}^{+\infty} \frac{g^{(k)}\left(\bar{a}_{0}\right)}{(k-1) !} \lambda^{k-1}\left\langle\epsilon,(\cdot)^{k-1}\right\rangle,
\end{gathered}
$$

where

$$
\begin{aligned}
& \tilde{w}=\left\{\lambda, \tilde{z}, \tilde{a}_{1}, \tilde{b}_{1}\right\}, \quad \tilde{z}=\{\tilde{a}, \tilde{b}\}=\left(\left\{\tilde{a}_{2}, \tilde{b}_{2}\right\}, \cdots,\left\{\tilde{a}_{N}, \tilde{b}_{N}\right\}, \tilde{a}_{0}^{\prime}\right), \\
& (\cdot)=\left(\lambda \sum_{l=2}^{N}\left(\tilde{a}_{l} e_{1}(l q)+\tilde{b}_{l} e_{2}(l q)\right)+\lambda \tilde{a}_{0}^{\prime} \epsilon+\tilde{a}_{1} e_{1}(q)+\tilde{b}_{1} e_{2}(q)\right) .
\end{aligned}
$$

Let's consider the norm

$$
|\tilde{z}|=\max _{2 \leqslant l \leqslant N}\left\{\left|\tilde{a}_{l}\right|,\left|\tilde{b}_{l}\right|,\left|\tilde{a}_{0}^{\prime}\right|\right\}
$$

and the radius $A$ ball-centred at the point

$$
\tilde{z}_{0}=\left(\left\{\tilde{a}_{2}^{0}, \tilde{b}_{2}^{0}\right\}, \cdots,\left\{\tilde{a}_{N}^{0}, \tilde{b}_{N}^{0}\right\}, \tilde{a}_{0}^{0}\right)
$$

in a set of vectors $\tilde{z}$, where

$$
\begin{aligned}
\tilde{a}_{l}^{0} & =\frac{T}{\lambda^{2}+\Phi(l q)-\Phi(q)} \sum_{k=3}^{+\infty} \frac{g^{(k)}\left(\bar{a}_{0}\right)}{(k-1) !} \lambda^{k-3}\left\langle\epsilon,\left(\tilde{a}_{1} e_{1}(q)+\tilde{b}_{1} e_{2}(q)\right)^{k-1} e_{1}(l q)\right\rangle, \\
\tilde{b}_{l}^{0} & =\frac{T}{\lambda^{2}+\Phi(l q)-\Phi(q)} \sum_{k=3}^{+\infty} \frac{g^{(k)}\left(\bar{a}_{0}\right)}{(k-1) !} \lambda^{k-3}\left\langle\epsilon,\left(\tilde{a}_{1} e_{1}(q)+\tilde{b}_{1} e_{2}(q)\right)^{k-1} e_{2}(l q)\right\rangle, \\
\tilde{a}_{0}^{\prime 0} & =\frac{T}{2\left(\lambda^{2}+\Phi(0)-\Phi(q)\right)} \sum_{k=3}^{+\infty} \frac{g^{(k)}\left(\bar{a}_{0}\right)}{(k-1) !} \lambda^{k-3}\left\langle\epsilon,\left(\tilde{a}_{1} e_{1}(q)+\tilde{b}_{1} e_{2}(q)\right)^{k-1}\right\rangle .
\end{aligned}
$$

Let $F(\tilde{w})$ be a nonlinear operator given by the right-hand part of system (16), then this system turns into

$$
\tilde{z}=F(\tilde{w})
$$


Theorem 1. Let

$$
\begin{gathered}
\min _{l}|\Phi(l q)-\Phi(q)|=\delta>0, \\
l=0,2,3,4, \cdots, N-1, N,
\end{gathered}
$$

and $\lambda_{0}>0$ satisfy the inequality

$\max _{l} \frac{T}{\left|\lambda^{2}+\Phi(l q)-\Phi(q)\right|}(2 N-1) \lambda \sum_{k=3}^{+\infty} \frac{\left|g^{(k)}\left(\bar{a}_{0}\right)\right|}{(k-2) !} \times \lambda^{k-3}((2 N-1) A \lambda+2 A)^{k-2}<1$,

then for all $\lambda \in\left[0, \lambda_{0}\right]$ and $\tilde{a}_{1}, \tilde{b}_{1}, h$ such that

$$
\tilde{a}_{1}^{2}+\tilde{b}_{1}^{2} \leqslant A^{2}, \quad|h|<|\Phi(0)|,
$$

there exist critical temperature $T_{0}(h)$ and interval $\left(T_{0}(h)-\lambda^{2}, T_{0}(h)\right)$ on which the solution of nonlinear equations (17) exists, being a continuously differentiable function of $\lambda \in\left[0, \lambda_{0}\right]$ and $\tilde{a}_{1}, \tilde{b}_{1}$ for $\tilde{a}_{1}^{2}+\tilde{b}_{1}^{2} \leqslant A^{2}$.

Proof. Operator $F$ maps radius $A$ ball-centred at the point

$$
z_{0}=F\left(\lambda,\{0,0\}, \tilde{a}_{1}, \tilde{b}_{1}\right)
$$

into itself if the inequality

$$
\left\|F\left(\lambda,\{\tilde{a}, \tilde{b}\}, \tilde{a}_{1}, \tilde{b}_{1}\right)-F\left(\lambda,\{0,0\}, \tilde{a}_{1}, \tilde{b}_{1}\right)\right\| \leqslant \alpha A, \quad \alpha<1,
$$

holds. Let's estimate the difference

$$
\begin{gathered}
\left\|F_{l}^{(p)}\left(\lambda,\{\tilde{a}, \tilde{b}\}, \tilde{a}_{1}, \tilde{b}_{1}\right)-F_{l}^{(p)}\left(\lambda,\{0,0\}, \tilde{a}_{1}, \tilde{b}_{1}\right)\right\| \\
\leqslant \frac{T}{\left|\lambda^{2}+\Phi(l q)-\Phi(q)\right|} \sum_{k=3}^{+\infty} \frac{\left|g^{(k)}\left(\bar{a}_{0}\right)\right|}{(k-1) !} \lambda^{k-3} \\
\times\left|\left\langle\epsilon,\left((\cdot)^{k-1}-\left(\tilde{a}_{1} e_{1}(q)+\tilde{b}_{1} e_{2}(q)\right)^{k-1}\right) e_{p}(l q)\right\rangle\right|, \\
p=0,1,2, \quad l=0, \overline{2, N} .
\end{gathered}
$$

From this

$$
\begin{aligned}
& \left\|F_{l}^{(p)}\left(\lambda,\{\tilde{a}, \tilde{b}\}, \tilde{a}_{1}, \tilde{b}_{1}\right)-F_{l}^{(p)}\left(\lambda,\{0,0\}, \tilde{a}_{1}, \tilde{b}_{1}\right)\right\| \\
& \leqslant \max _{l} \frac{T}{\left|\lambda^{2}+\Phi(l q)-\Phi(q)\right|} \sum_{k=3}^{+\infty} \frac{\left|g^{(k)}\left(\bar{a}_{0}\right)\right|}{(k-1) !} \lambda^{k-3} \\
& \times \lambda(2 N-1) A \sum_{i=0}^{k-2}(\lambda(2 N-1) A)^{k-2-i}(2 A)^{i} \\
& \leqslant \max _{l} \frac{T}{\left|\lambda^{2}+\Phi(l q)-\Phi(q)\right|} \lambda(2 N-1) A
\end{aligned}
$$




$$
\times \sum_{k=3}^{+\infty} \frac{\left|g^{(k)}\left(\bar{a}_{0}\right)\right|}{(k-2) !} \lambda^{k-3}(\lambda(2 N-1) A+2 A)^{k-2} .
$$

At last one can estimate the difference

$$
\begin{aligned}
& \left\|F\left(\lambda,\left\{\tilde{a}^{\prime}, \tilde{b}^{\prime}\right\}, \tilde{a}_{1}, \tilde{b}_{1}\right)-F\left(\lambda,\{\tilde{a}, \tilde{b}\}, \tilde{a}_{1}, \tilde{b}_{1}\right)\right\| \leqslant \max _{l} \frac{T}{\left|\lambda^{2}+\Phi(l q)-\Phi(q)\right|} \\
& \times \sum_{k=3}^{+\infty} \frac{\left|g^{(k)}\left(\bar{a}_{0}\right)\right|}{(k-2) !} \lambda^{k-3} \lambda(2 N-1)(\lambda(2 N-1) A+2 A)^{k-2}\left\|\left\{\tilde{a}^{\prime}, \tilde{b}^{\prime}\right\}-\{\tilde{a}, \tilde{b}\}\right\| .
\end{aligned}
$$

Therefore, choosing a rather small $\lambda$ we realize the inequality

$$
\begin{gathered}
\max _{l} \frac{T}{\left|\lambda^{2}+\Phi(l q)-\Phi(q)\right|}(2 N-1) \lambda \sum_{k=3}^{+\infty} \frac{\left|g^{(k)}\left(\bar{a}_{0}\right)\right|}{(k-2) !} \\
\times \lambda^{k-3}((2 N-1) A \lambda+2 A)^{k-2}<1,
\end{gathered}
$$

that guarantees inequality (18) and contractivity conditions for nonlinear map $F$. If $\tilde{a}_{1}$ and $\tilde{b}_{1}$ are such that $\tilde{a}_{1}^{2}+\tilde{b}_{1}^{2} \leqslant A^{2}$, then inequality (18) holds. This ends the proof.

The similar theorem is valid for rational $q$.

Remark 1. Continuous differentiability with respect to the variables $\lambda$ and $\tilde{a}_{1}, \tilde{b}_{1}$ results from the continuous differentiability of the successive approximations and their uniform convergence to the solution.

Theorem 2. For all the irrational $q$ that guarantee the validity of

$$
\begin{gathered}
\min _{l}|\Phi(l q)-\Phi(q)|=\delta>0, \\
l=0,2,3,4, \cdots, N-1, N,
\end{gathered}
$$

there is such $\lambda_{1}>0$, that system (10) is solvable for $0<\lambda<\lambda_{1}$, $|h|<|\Phi(0)|$. The action of group $G(5)$ on this solution gives different solutions of system (10) being continuously differentiable functions of $\lambda$.

Proof. Having calculated the averages,

$$
I_{s, m}=\left\langle\prod_{k=1}^{s} e_{1}\left(i_{k} q\right) \prod_{l=1}^{m} e_{2}\left(j_{l} q\right), \epsilon\right\rangle
$$

entering equations (13) by formula [5]

$$
I_{s, m}=\left\{\begin{array}{c}
0, \quad m=2 M+1, \quad M=0,1,2, \cdots, \\
(-1)^{M} 2^{-s-m} \sum_{\substack{t_{k^{\prime}}= \pm i_{k^{\prime}} \\
\tau_{l}= \pm j_{l}}} \delta_{t_{1}+\cdots+t_{s}+\tau_{1}+\cdots+\tau_{m}, 0}(-1)^{k}, m=2 M
\end{array}\right.
$$


where $k$ is the number of negative $\tau_{l}$, we write system (13) as

$$
\begin{aligned}
& \tilde{a}_{1}=T\left(\frac{g^{(3)}\left(\bar{a}_{0}\right)}{2}\left(\frac{\tilde{a}_{2} \tilde{a}_{1}+\tilde{b}_{2} \tilde{b}_{1}}{2}+\tilde{a}_{0}^{\prime} \tilde{a}_{1}\right)+\frac{g^{(4)}\left(\bar{a}_{0}\right)}{16}\left(\tilde{a}_{1}^{3}+\tilde{a}_{1} \tilde{b}_{1}^{2}\right)+\lambda^{2} \tilde{\varphi}_{1}^{(1)}(\tilde{w})\right), \\
& \tilde{b}_{1}=T\left(\frac{g^{(3)}\left(\bar{a}_{0}\right)}{2}\left(\frac{\tilde{b}_{2} \tilde{a}_{1}-\tilde{a}_{2} \tilde{b}_{1}}{2}+\tilde{a}_{0}^{\prime} \tilde{b}_{1}\right)+\frac{g^{(4)}\left(\bar{a}_{0}\right)}{16}\left(\tilde{b}_{1}^{3}+\tilde{b}_{1} \tilde{a}_{1}^{2}\right)+\lambda^{2} \tilde{\varphi}_{1}^{(2)}(\tilde{w})\right),
\end{aligned}
$$

where

$$
\begin{aligned}
g^{(3)}\left(\bar{a}_{0}\right) & =\frac{4 \bar{a}_{0}}{\left(1-\bar{a}_{0}^{2}\right)^{2}}, \\
g^{(4)}\left(\bar{a}_{0}\right) & =\frac{4}{\left(1-\bar{a}_{0}^{2}\right)^{2}}+\frac{16 \bar{a}_{0}^{2}}{\left(1-\bar{a}_{0}^{2}\right)^{3}} .
\end{aligned}
$$

For the zero approximation, solutions for $\tilde{a}_{0}^{\prime}, \tilde{a}_{2}$ and $\tilde{b}_{2}$ are

$$
\begin{aligned}
\tilde{a}_{0}^{\prime 0} & =\frac{T g^{(3)}\left(\bar{a}_{0}\right)\left(\tilde{a}_{1}^{2}+\tilde{b}_{1}^{2}\right)}{8\left(\lambda^{2}+\Phi(0)-\Phi(q)\right)}, \\
\tilde{a}_{2}^{0} & =\frac{T g^{(3)}\left(\bar{a}_{0}\right)\left(\tilde{a}_{1}^{2}-\tilde{b}_{1}^{2}\right)}{8\left(\lambda^{2}+\Phi(2 q)-\Phi(q)\right)}, \\
\tilde{b}_{2}^{0} & =\frac{T g^{(3)}\left(\bar{a}_{0}\right) \tilde{a}_{1} \tilde{b}_{1}}{4\left(\lambda^{2}+\Phi(2 q)-\Phi(q)\right)} .
\end{aligned}
$$

Due to the continuous transformation group $G(5)$, the branching equation has the form [7]

$$
T\left(D\left(\tilde{a}_{1}^{2}+\tilde{b}_{1}^{2}\right)+\lambda^{2} \tilde{\varphi}_{0}\left(\lambda, \tilde{a}_{1}^{2}+\tilde{b}_{1}^{2}\right)\right)=1
$$

where

$$
\begin{aligned}
D= & \frac{T \bar{a}_{0}^{2}}{\left(1-\bar{a}_{0}^{2}\right)^{4}}\left(\frac{1}{2\left(\lambda^{2}+\Phi(2 q)-\Phi(q)\right)}+\frac{2}{\lambda^{2}+\Phi(0)-\Phi(q)}\right) \\
& +\frac{\bar{a}_{0}^{2}}{\left(1-\bar{a}_{0}^{2}\right)^{3}}+\frac{1}{4\left(1-\bar{a}_{0}^{2}\right)^{2}} .
\end{aligned}
$$

In terms of $u=\tilde{a}_{1}^{2}+\tilde{b}_{1}^{2}$ equation (19) becomes

$$
u=\left(1 / T-\lambda^{2} \tilde{\varphi}_{0}(\lambda, u)\right) / D,
$$

where $\tilde{\varphi}_{0}(\lambda, u)$ is a continuously differentiable function of all the variables in the vicinity of

$$
|\lambda|<\lambda_{0}, \quad 0 \leqslant u \leqslant 2 A^{2} .
$$

The inequalities

$$
\begin{aligned}
& \sup _{0 \leqslant u \leqslant 2 A^{2}} D^{-1}\left|T^{-1}-\lambda^{2} \tilde{\varphi}_{0}(\lambda, u)\right|<2 A^{2}, \\
& \sup _{0 \leqslant u \leqslant 2 A^{2}} \lambda^{2} D^{-1}\left|\partial \tilde{\varphi}_{0}(\lambda, u) / \partial u\right|<1
\end{aligned}
$$


hold for a rather small $\lambda$ and $2 A^{2}>1 / T D$. From inequalities (21) it follows that equation (20) has the solution, which is a continuously differentiable function of $\lambda$ for rather small $\lambda<\lambda_{1}$. This ends the proof.

Theorem 3. For those rational $q$, for which $N_{1} \neq 3$, there is $\lambda_{0}>0$ such that a nonzero solution to system (9) exists for $0<\lambda<\lambda_{0}$,

$|h|<|\Phi(0)|$. The action of group (3) on this solution gives different solutions to system (9) being continuously differentiable functions of $\lambda$.

Proof. Similarly to the case of irrational $q$, one can prove that the problem of the existence of a nonzero solution to system (9) is equivalent to that of branching equation [8]. To derive branching equations for different values of period $N_{1}$ we calculate the averages $I_{s, m}$ entering system (6) by formula [5]

$$
I_{s, m}=\left\{\begin{array}{c}
0, \quad m=2 M+1, \quad M=0,1,2, \cdots, \\
(-1)^{M} 2^{-s-m} \sum_{r=0, \pm 1, \pm 2, \cdots} \sum_{\substack{t_{k^{\prime}}= \pm i_{k^{\prime}} \\
\tau_{l}= \pm j_{l}}} \delta_{t_{1}+\cdots+t_{s}+\tau_{1}+\cdots+\tau_{m},|r| N_{1}}(-1)^{k}, m=2 M
\end{array}\right.
$$

where $k$ is the number of negative $\tau_{l}$.

For $N_{1}=2$ we obtain a single branching equation

$$
\tilde{a}_{1}=T\left(g^{(4)}\left(\bar{a}_{0}\right) \tilde{a}_{1}^{3} / 6+\lambda^{2} \tilde{\varphi}_{1}^{(2)}\left(\lambda, \tilde{a}_{1}^{2}\right) \tilde{a}_{1}\right) / 2 .
$$

There are small solutions of equation (22) which are continuously differentiable functions of $\lambda$.

For $N_{1}=3$ the branching equations are

$$
\begin{aligned}
\tilde{a}_{1}= & T\left(\tilde{a}_{1}\left(\tilde{a}_{1}^{2}+\tilde{b}_{1}^{2}\right)\left(\left(\frac{g^{(3)}\left(\bar{a}_{0}\right)}{4}\right)^{2} \frac{T}{\lambda^{2}+\Phi(0)-\Phi(q)} \frac{g^{(4)}\left(\bar{a}_{0}\right)}{16}\right)\right. \\
& \left.+\frac{g^{(3)}\left(\bar{a}_{0}\right)}{8 \lambda}\left(\tilde{a}_{1}^{2}-\tilde{b}_{1}^{2}\right)+\lambda \varphi_{1}^{(3)}\left(\lambda, \tilde{a}_{1}, \tilde{b}_{1}^{2}\right)\right) \\
\tilde{b}_{1}= & T\left(\tilde{b}_{1}\left(\tilde{a}_{1}^{2}+\tilde{b}_{1}^{2}\right)\left(\left(\frac{g^{(3)}\left(\bar{a}_{0}\right)}{4}\right)^{2} \frac{T}{\lambda^{2}+\Phi(0)-\Phi(q)}+\frac{g^{(4)}\left(\bar{a}_{0}\right)}{16}\right)\right. \\
& \left.-\frac{g^{(3)}\left(\bar{a}_{0}\right)}{4 \lambda} \tilde{b}_{1} \tilde{a}_{1}+\lambda \varphi_{2}^{(3)}\left(\lambda, \tilde{a}_{1}, \tilde{b}_{1}^{2}\right) \tilde{b}_{1}\right) .
\end{aligned}
$$

System (23) has no small solutions for a rather small $\lambda$. As a result, branching from the paramagnetic phase to the commensurate one of period $N_{1}=3$ does not occur in the magnetic field.

For $N_{1}=4$ (antiphase) the branching equations are

$$
\tilde{a}_{1}=T\left[\tilde{a}_{1}\left(\frac{g^{(3)}\left(\bar{a}_{0}\right)}{2}\right)^{2} \frac{T}{2}\left(\frac{\tilde{a}_{1}^{2}-\tilde{b}_{1}^{2}}{\lambda^{2}+\Phi(2 q)-\Phi(q)}+\frac{\tilde{a}_{1}^{2}+\tilde{b}_{1}^{2}}{2\left(\lambda^{2}+\Phi(0)-\Phi(q)\right)}\right)\right.
$$




$$
\begin{aligned}
& \left.+\frac{g^{(4)}\left(\bar{a}_{0}\right)}{12} \tilde{a}_{1}^{3}+\lambda^{2} \varphi_{1}^{(4)}\left(\lambda, \tilde{a}_{1}, \tilde{b}_{1}^{2}\right)\right], \\
\tilde{b}_{1}= & T\left[\tilde{b}_{1}\left(\frac{g^{(3)}\left(\bar{a}_{0}\right)}{2}\right)^{2} \frac{T}{2}\left(\frac{\tilde{a}_{1}^{2}+\tilde{b}_{1}^{2}}{2\left(\lambda^{2}+\Phi(0)-\Phi(q)\right)}-\frac{\tilde{a}_{1}^{2}-\tilde{b}_{1}^{2}}{\lambda^{2}+\Phi(2 q)-\Phi(q)}\right)\right. \\
& \left.+\frac{g^{(4)}\left(\bar{a}_{0}\right)}{12} \tilde{b}_{1}^{3}+\lambda^{2} \varphi_{2}^{(4)}\left(\lambda, \tilde{a}_{1}, \tilde{b}_{1}^{2}\right) \tilde{b}_{1}\right] .
\end{aligned}
$$

For $N_{1}=5$ the branching equations are

$$
\begin{aligned}
& \tilde{a}_{1}=T\left[\tilde{a}_{1}\left(\tilde{a}_{1}^{2}+\tilde{b}_{1}^{2}\right) \tilde{D}+\lambda \varphi_{1}^{(5)}\left(\lambda, \tilde{a}_{1}, \tilde{b}_{1}^{2}\right)\right], \\
& \tilde{b}_{1}=T\left[\tilde{b}_{1}\left(\tilde{a}_{1}^{2}+\tilde{b}_{1}^{2}\right) \tilde{D}+\lambda \varphi_{2}^{(5)}\left(\lambda, \tilde{a}_{1}, \tilde{b}_{1}^{2}\right) \tilde{b}_{1}^{2}\right],
\end{aligned}
$$

where

$$
\tilde{D}=T\left(\frac{g^{(3)}\left(\bar{a}_{0}\right)}{4}\right)^{2}\left(\frac{1}{2\left(\lambda^{2}+\Phi(2 q)-\Phi(q)\right)}+\frac{1}{\lambda^{2}+\Phi(0)-\Phi(q)}\right)+\frac{g^{(4)}\left(\bar{a}_{0}\right)}{16} .
$$

If $N_{1} \geqslant 6$, then the branching equations are

$$
\begin{aligned}
& \tilde{a}_{1}=T\left[\tilde{a}_{1}\left(\tilde{a}_{1}^{2}+\tilde{b}_{1}^{2}\right) \tilde{D}+\lambda^{2} \varphi_{1}^{\left(N_{1}\right)}\left(\lambda, \tilde{a}_{1}, \tilde{b}_{1}^{2}\right)\right], \\
& \tilde{b}_{1}=T\left[\tilde{b}_{1}\left(\tilde{a}_{1}^{2}+\tilde{b}_{1}^{2}\right) \tilde{D}+\lambda^{2} \varphi_{2}^{\left(N_{1}\right)}\left(\lambda, \tilde{a}_{1}, \tilde{b}_{1}^{2}\right) \tilde{b}_{1}^{2}\right] .
\end{aligned}
$$

One essential distinction occurs, namely, the second equation allows a zero solution, but the first one assumes a nonzero solution with respect to $\tilde{a}_{1}$. We denote

$$
\tilde{\varphi}_{1}^{\left(N_{1}\right)}\left(t, \tilde{a}_{1}\right)=\frac{\varphi_{1}^{\left(N_{1}\right)}\left(t, \tilde{a}_{1}, 0\right)}{\tilde{a}_{1}}, \quad \tilde{\varphi}_{1}^{\left(N_{1}\right)}(t, 0)=0 .
$$

Considering $\tilde{a}_{1} \neq 0$ and dividing by it, we obtain the equation

$$
\tilde{a}_{1}= \pm \sqrt{\tilde{D}^{-1}\left(T^{-1}-\lambda^{2} \tilde{\varphi}_{1}^{\left(N_{1}\right)}\left(\lambda, \tilde{a}_{1}\right)\right)} .
$$

For $A>1 / \sqrt{\tilde{D} T}$ and a positive sign we have

$$
\tilde{a}_{1}=\sqrt{\tilde{D}^{-1}\left(T^{-1}-\lambda^{2} \tilde{\varphi}_{1}^{\left(N_{1}\right)}\left(\lambda, \tilde{a}_{1}\right)\right)} .
$$

From the conditions

$$
\begin{gathered}
\sup _{\tilde{a}_{1} \leqslant A} \sqrt{\tilde{D}^{-1}\left(T^{-1}-\lambda^{2} \tilde{\varphi}_{1}^{\left(N_{1}\right)}\left(\lambda, \tilde{a}_{1}\right)\right)} \leqslant A, \\
\sup _{\tilde{a}_{1} \leq A} \frac{\lambda^{2} T\left|\frac{d}{d \tilde{a}_{1}} \tilde{\varphi}_{1}^{\left(N_{1}\right)}\left(\lambda, \tilde{a}_{1}\right)\right|}{\tilde{D} \sqrt{\tilde{D}^{-1}\left(T^{-1}-\lambda^{2} \tilde{\varphi}_{1}^{\left(N_{1}\right)}\left(\lambda, \tilde{a}_{1}\right)\right)}}<1
\end{gathered}
$$


it follows that (28) has a unique solution for rather small $0<\lambda<\lambda_{0}$.

Taking the minus sign in equation (27), one obtains an alternate nonzero solution.

\section{References}

1. Villain J. La structure des substances magnetiques. // J. Phys. Chem. Solids, 1959, vol. 11, p. 303-309.

2. Kaplan T.A. Classical spin-configuration stability in the presence of competing exchange forces. // Phys. Rev., 1959, vol. 116, p. 888-889.

3. Gonchar N.S., Kozyrski W.H. Hidden symmetry and small branchings in the anisotropic model. // Ukr. Math. J., 1991, vol. 43, No 11, p. 1509-1516 (in Ukrainian).

4. Gonchar N.S., Hajduk H.G., Kozyrski W.H. Branching equations for commensurate phase of the ANNNI model. Preprint ITP-95-2U, Kiev, 1995 (in Ukrainian).

5. Gonchar N.S., Kozyrski W.H. Incommensurate phases. Preprint ITP-90-51R, Kiev, 1990 (in Russian).

6. Gonchar N.S., Kozyrski W.H. One-parameter symmetry group of incommensurate phases of the ANNNI model. // Ukr. Fiz. Zhurn., 1991, vol. 36, No 12, p. 1857-1864 (in Ukrainian).

7. Gonchar N.S., Hajduk H.G. Phase transition in the ANNNI model in the presence of external magnetic field. Preprint ITP-94-31U, Kiev, 1994 (in Ukrainian).

8. Hajduk H.G. Branching equations for commensurate phases of the ANNNI model in the presence of external magnetic field. Preprint ITP-95-22U, Kiev, 1995 (in Ukrainian). 


\title{
Модульовані структури поблизу критичності в моделі ANNNI з зовнішнім магнітним полем
}

\author{
М.С.Гончар, І.Г.Гайдук
}

Відділ математичного моделювання Інституту теоретичної фізики ім. М.М.Боголюбова НАН України, 252143 Київ, вул. Метрологічна $14^{\text {б }}$

Отримано 3 грудня 1997 р.

Знайдено умови фазового переходу із парамагнітного стану до модульованої структури в класі анізотропних моделей Ізінга з зовнішнім магнітним полем. Обчислено значення критичного магнітного поля. Отримані рівняння галуження та доведені теореми про малі галуження для співмірних та неспівмірних конфігурацій.

Ключові слова: анізотропна модель Ізінга, фазовий перехід, парамагнітна фаза, співмірна конфігурація, неспівмірна конфігурація, критичне магнітне поле

PACS: $05.50 .+q, 64.60 . C n$ 